\title{
APPROXIMATE CONTROLLABILITY OF SEMILINEAR FRACTIONAL STOCHASTIC SYSTEM WITH NONLOCAL CONDITIONS
}

\author{
URVASHI ARORA ${ }^{1}$, N. SUKAVANAM ${ }^{2}$ \\ ${ }^{1}$ Department of Mathematics \\ Bennett University \\ Greater Noida, INDIA \\ ${ }^{2}$ Department of Mathematics \\ Indian Institute of Technology \\ Roorkee, INDIA
}

\begin{abstract}
This paper deals with the approximate controllability of semilinear fractional stochastic system of order $\rho \in(1,2]$ with nonlocal conditions. By using Sadovskii's fixed point theorem with fractional calculus and stochastic analysis theory, we derive a new set of sufficient conditions for the approximate controllability of fractional stochastic system with nonlocal conditions under the assumption that the corresponding linear system is approximately controllable. Finally, an application to a fractional stochastic system with nonlocal initial conditions is provided to illustrate the feasibility of the obtained results.
\end{abstract}

AMS Subject Classification: 93B05, 26A33

Received: April 1, 2017; Accepted: Ocotber 14, 2017;

Published: January 4, 2018 doi: 10.12732/dsa.v27i1.3

Dynamic Publishers, Inc., Acad. Publishers, Ltd. https://acadsol.eu/dsa

\section{INTRODUCTION}

In the recent years, the investigation of fractional differential equation has been picking up much attention of researchers. This is due to the fact that fractional differential equations have various applications in engineering and scientific disciplines, for example, fluid dynamics, fractal theory, diffusion in porous media, fractional biological neurons, traffic flow, polymer rheology, neural network modeling, viscoelastic 
panel in super sonic gas flow, real system characterized by power laws, electrodynamics of complex medium, sandwich system identification, nonlinear oscillation of earthquake, models of population growth, mathematical modeling of the diffusion of discrete particles in a turbulent fluid, nuclear reactors and theory of population dynamics. Fractional calculus is the generalization of the ordinary differentiation and integration to arbitrary non integer order. The fractional differential equation is an important tool to describe the memory and hereditary properties of various materials and phenomena. For more details on fractional calculus see [2, 10, 19, 20].

On the other hand, control problems have attracted many physicists, engineers and mathematicians and notable contributions have been made to both theory and applications. The notion of controllability has played a central role throughout the history of modern control theory. This is the qualitative property of control systems and is of particular importance in control theory. Many dynamical systems are such that the control does not affect the complete state of the dynamical system but only a part of it. On the other hand, very often in real industrial processes it is possible to observe only a certain part of the complete state of the dynamical system. Therefore, it is very important to determine whether or not control of the complete state of the dynamical system is possible. So, here the concept of complete controllability and approximate controllability arises. Roughly speaking, controllability generally means that it is possible to steer the dynamical system from an arbitrary initial state to the desired final state using the set of admissible controls. Controllability is also strongly connected with the theory of minimal realization of linear time-invariant control systems. Conceived by Kalman [9], controllability study was started systematically at the beginning of the sixties. Since then various researches have been carried out extensively in the context of finite -dimensional deterministic linear systems, nonlinear systems and infinite dimensional systems using different kinds of approaches. Controllability of deterministic systems is widely used in many fields of science and technology.

On the other hand, noise or stochastic perturbation is unavoidable and omnipresent in nature as well as in man-made systems, so we have to move from deterministic models to stochastic models. Stochastic differential equations play an important role in formulation and analysis of mechanical, electrical, control engineering, and physical sciences. Motivated by these facts many researchers are showing great interest to establish an appropriate system to investigate qualitative properties such as existence, uniqueness, controllability and stability of these physical processes with the help of fractional calculus, Stochastic analysis and fixed point theorems.

Klamka [12] studied the controllability of linear stochastic systems in finite dimensional spaces with delay and without delay in control. In [11], Klamka and Socha discussed about the controllability of stochastic systems. Mahmudov et al. [17, 16] 
established results for controllability of linear and semilinear stochastic systems in Hilbert spaces. Shen and Sun [15] studied the controllability of stochastic first order nonlinear systems with delay in control in finite dimensional as well as in infinite dimensional spaces.

On the other hand, non local conditions introduced by Byszewski et al. [4] are the conditions given by an expression where the value of an unknown function is expressed by the value(s) of this function at regular intervals rather than continuously over the history period. He has done a great work on nonlocal condition problems and claim that these types of conditions are usually more precise for physical measurements than the classical ones as more information was taken into account at the oneset of the experiment. In recent times, many researchers have studied the existence and uniqueness of the mild solution of semilinear systems with nonlocal conditions.

Kumar.S and Sukavanam [13] established sufficient conditions for the controllability of second order deterministic systems with nonlocal conditions in Banach spaces using Sadovskii's Fixed point theorem. Shukla et al.[14] established sufficient conditions for the approximate controllability of retarded semilinear stochastic system with nonlocal conditions by assuming that the corresponding linear system is approximately controllable. Arora and Sukavanam [1] established the controllability of second order semilinear stochastic system with nonlocal conditions using Sadovskii's Fixed Point theorem. Very less papers deal with the controllability of fractional stochastic systems of order $\rho \in(1,2]$. Farahi and Guendouzi [7] studied the approximate controllability of fractional neutral stochastic evolution equations of order $\rho \in(0,1]$ with nonlocal conditions using Sadovskii's Fixed Point theorem. In [8], Guendouzi and Farahi established results on the aproximate controllability of semilinear fractional stochastic dynamic systems with nonlocal conditions in Hilbert Spaces using Schefer's Fixed point theorem.

To the best of our knowledge, the existing articles in the literature concentrate to examine the existence, uniqueness and controllability of mild solutions for fractional stochastic systems of order $\rho \in(1,2]$ in Hilbert space $\mathrm{H}$ using Banach Fixed Point Theorem, Schefer's Fixed Point theorem. there is no work reported on the controllability of fractional stochastic system of order $\rho \in(1,2]$ using Sadovskii's Fixed Point Theorem. Motivated by the above analysis, in this paper we establish sufficient conditions for the approximate controllability of fractional stochastic system of order $\rho \in(1,2]$ with nonlocal conditions using Sadovskii's Fixed Point Theorem.

\section{PROBLEM FORMULATION AND PRELIMINARIES}

Let $\mathcal{Y}, U$ and $\mathcal{K}$ be the separable Hilbert spaces. Let $(\Omega, \Im, \mathbb{P})$ be a complete probability space equipped with a normal filtration $\Im_{t}, t \in J=[0, b]$ such that the filtration 
$\Im_{t}$ is a right continuous increasing family and $\Im_{0}$ contains all $\mathbb{P}$-null sets. Let $\omega$ be a $Q$-Wiener process on $\left(\Omega, \Im_{t}, \mathbb{P}\right)$ with the covariance operator $Q$ such that $\operatorname{tr} Q<\infty$. A $\mathcal{Y}$-valued random variable is a $\Im$ measurable function $y(t): \Omega \rightarrow \mathcal{Y}$ and the collection of random variables $S=\{y(t): \Omega \rightarrow \mathcal{Y} \mid t \in[0, b]\}$ is called a stochastic process. We assume that there exists a complete orthonormal system $e_{k}$ in $\mathcal{K}$, a bounded sequence of nonnegative real numbers $\lambda_{k}$ such that $Q e_{k}=\lambda_{k} e_{k}, k=1,2, \cdots$ and a sequence $B_{k}$ of independent Brownian motions such that

$$
<w(t), e>=\sum_{k=1}^{\infty} \sqrt{\lambda_{k}}<e_{k}, e>B_{k}(t), \quad e \in \mathcal{K}, t \in J
$$

and $\Im_{t}=\Im_{t}^{\omega}$, where $\Im_{t}^{\omega}$ is the $\sigma$-algebra generated by $\omega$. In order to define stochastic integrals with respect to the $Q$-Wiener process $\omega(t)$, we introduce the subspace $\mathcal{K}_{0}=Q^{1 / 2}(\mathcal{K})$ of $\mathcal{K}$ which is endowed with the inner product $\left(y_{1}, y_{2}\right)_{\mathcal{K}_{0}}=$ $\left(Q^{-1 / 2} y_{1}, Q^{-1 / 2} y_{2}\right)$ and is a Hilbert space. Let $\mathcal{L}_{2}{ }^{0}=\mathcal{L}_{2}\left(Q^{1 / 2} \mathcal{K} ; \mathcal{Y}\right)$ be the space of all Hilbert-Schmidt operators from $Q^{1 / 2} \mathcal{K}$ to $\mathcal{Y}$ with the norm

$$
\|\varphi\|_{\mathcal{L}_{2}^{0}}^{2}=\operatorname{tr}\left(\left(\varphi Q^{1 / 2}\right)\left(\varphi Q^{1 / 2}\right)^{*}\right),
$$

for $\varphi \in \mathcal{L}_{2}^{0}$. Clearly, for any bounded linear operator $\varphi \in \mathcal{L}(\mathcal{K}, \mathcal{Y})$, this norm reduces to

$$
\|\varphi\|_{\mathcal{L}_{2}^{0}}^{2}=\operatorname{tr}\left(\varphi Q \varphi^{*}\right)=\sum_{k=1}^{\infty}\left\|\sqrt{\lambda_{k}} \varphi \zeta_{k}\right\|^{2},
$$

Let $L_{2}\left(\Omega, \Im_{b}, \mathcal{Y}\right)$ be the Banach space of $\Im_{b}$ measurable square integrable random variables with values in the Hilbert space $\mathcal{Y}$. Let $L_{\Im}^{2}(J, \mathcal{Y})$ be the space of all $\Im_{t}$-adapted, $\mathcal{Y}$-valued measurable square integrable processes on $J \times \Omega$. Let $C\left([0, b] ; L_{2}\left(\Omega, \Im_{t}, \mathcal{Y}\right)\right)$ be the Banach space of continuous maps from $[0, b]$ into $L_{2}\left(\Omega, \Im_{t}\right.$, $\mathcal{Y})$ satisfying the condition $\sup _{t \in J} \mathbb{E}\|x(t)\|^{2}<\infty$.

Let $\mathcal{Y}_{2}$ be the closed subspace of $C\left([0, b] ; L_{2}\left(\Omega, \Im_{t}, \mathcal{Y}\right)\right)$ consisting of measurable and $\Im_{t}$ - adapted $\mathcal{Y}$ valued processes $\phi \in C\left([0, b] ; L_{2}\left(\Omega, \Im_{t}, \mathcal{Y}\right)\right)$ endowed with the norm

$$
\|\phi\|_{\mathcal{Y}_{2}}=\left(\sup _{t \in[0, b]} \mathbb{E}\|\phi(t)\|_{\mathcal{Y}}^{2}\right)^{1 / 2}
$$

where $\mathbb{E}$ is defined as integration with respect to probability measure $\mathbb{P}$.

In this paper, we consider a mathematical model given by the following semilinear fractional stochastic system of order $\rho \in(1,2]$ with nonlocal conditions of the form

$$
\left.\begin{array}{rl}
{ }^{c} D_{t}^{\rho} x(t) & =[A x(t)+B u(t)+\mathfrak{f}(t, x(t))]+\sigma(t, x(t)) \frac{d \omega(t)}{d t}, \quad t \in J \\
x(0) & =x_{0}+g(x), \quad x^{\prime}(0)=x_{1}+g_{1}(x)
\end{array}\right\}
$$

where $\rho \in(1,2],{ }^{c} D_{t}^{\rho}$ is the caputo fractional derivative and the positive constant $b<\infty$. Here, the state $x($.) takes values in a Banach space $\mathcal{Y}$ and the control function 
$u \in L_{\Im}^{2}(J, U)$; a Banach space of admissible control functions. $A$ is the infinitesimal generator of strongly continuous $\rho$-order cosine family $\left\{C_{\rho}(t)\right\}$ on the Banach space $\mathcal{Y}$. $B$ is a bounded linear operator from the Hilbert space $U$ into $\mathcal{Y}$. The maps $\mathfrak{f}: J \times \mathcal{Y} \rightarrow \mathcal{Y} ; \sigma: J \times \mathcal{Y} \rightarrow L_{2}^{0}$ are nonlinear suitable functions; $x_{0}$ and $x_{1}$ are $\Im_{0}$ measurable $\mathcal{Y}$ valued random variables independent of $\omega ; g, g_{1}$ are continuous functions from $C(J, \mathcal{Y}) \rightarrow \mathcal{Y}$.

For simplicity of considerations, we generally assume that the set of admissible controls is $U_{a d}=L_{\Im}^{2}(J, U)$.

Now, we discuss some basic concepts of fractional calculus, stochastic integral and semigroup theory of linear operators.

Here $C([0, b] ; \mathcal{Y})$ and $C^{1}([0, b] ; \mathcal{Y})$ denote the space of functions which are continuous and 1-time continuously differentiable, respectively.

Definition 2.1. [10]. If $x(t) \in L_{1}([0, b] ; \mathcal{Y})$, then the Riemann-liouville fractional integral of order $\rho>0$ is defined by

$$
J_{t}^{\rho} x(t)=\frac{1}{\Gamma(\rho)} \int_{0}^{t}(t-s)^{\rho-1} x(s) d s
$$

where $\Gamma(\rho)$ is the Gamma function defined as $\Gamma(\rho)=\int_{0}^{\infty} e^{-t} t^{\rho-1} d t$.

Definition 2.2. [10]. The Riemann- liouville fractional derivative of a function $x(t) \in L_{1}([0, b] ; \mathcal{Y})$ of order $\rho \in(1,2]$ is defined by

$$
D_{t}^{\rho} x(t)=D^{2} J_{t}^{2-\rho} x(t)=\frac{1}{\Gamma(2-\rho)} \frac{d^{2}}{d t^{2}} \int_{0}^{t}(t-s)^{1-\rho} x(s) d s
$$

Definition 2.3. [10]. The Caputo fractional derivative of order $\rho \in(1,2]$ is defined by

$$
{ }^{c} D_{t}^{\rho} x(t)=J_{t}^{2-\rho} D^{2} x(t)=\frac{1}{\Gamma(2-\rho)} \int_{t_{0}}^{t}(t-s)^{1-\rho}\left[\frac{d^{2}}{d s^{2}} x(s)\right] d s,
$$

where $x(t) \in L_{1}([0, b] ; \mathcal{Y}) \cap C^{1}([0, b] ; \mathcal{Y})$.

Consider the following fractional order system:

$$
{ }^{c} D_{t}^{\rho} x(t)=A x(t), x(0)=\eta x^{\prime}(0)=0,
$$

where $\rho \in(1,2] ; A: D(A) \subseteq \mathcal{Y} \rightarrow \mathcal{Y}$ is a closed and densely defined operator in a Banach space $\mathcal{Y}$. Applying Riemann fractional integral of order $\rho$ on both sides of $(2.2)$, we get

$$
x(t)=\eta+\frac{1}{\Gamma(\rho)} \int_{0}^{t}(t-s)^{\rho-1} A x(s) d s
$$


Definition 2.4. [2]. Let $\rho \in(1,2]$. A family $\left\{C_{\rho}(t)\right\}_{t \geq 0} \subset \mathbb{L}(\mathcal{Y})$ is called a solution operator (or a strongly continuous $\rho$-order fractional cosine family) for (2.2) if the following conditions are satisfied:

1. $C_{\rho}(t)$ is strongly continuous for $t \geq 0$ and $C_{\rho}(0)=I$;

2. $C_{\rho}(t) D(A) \subset D(A)$ and $A C_{\rho}(t) \eta=C_{\rho}(t) A \eta$ for all $\eta \in D(A), t \geq 0$;

3. $C_{\rho}(t) \eta$ is a solution of $(2.2)$ for all $\eta \in D(A), t \geq 0$.

$A$ is called infinitesimal generator of $C_{\rho}(t)$. The strongly continuous $\rho$-order fractional cosine family is also called $\rho$-order cosine family.

Definition 2.5. The fractional sine family $S_{\rho}:[0, \infty) \rightarrow \mathbb{L}(\mathcal{Y})$ associated with $C_{\rho}$ is defined by

$$
S_{\rho}(t)=\int_{0}^{t} C_{\rho}(s) d s, \quad t \geq 0
$$

Definition 2.6. The fractional Riemann-Liouville family $P_{\rho}:[0, \infty) \rightarrow \mathbb{L}(\mathcal{Y})$ associated with $C_{\rho}$ is defined by

$$
P_{\rho}(t)=J_{t}^{\rho-1} C_{\rho}(t)
$$

Definition 2.7. The $\rho$-order cosine family $C_{\rho}(t)$ is called exponentially bounded if there are constants $M \geq 1$ and $\omega \geq 0$ such that

$$
\left\|C_{\rho}(t)\right\| \leq M e^{\omega t}, t \geq 0
$$

An operator $A$ is said to belong to $C^{\rho}(\mathcal{Y}, M, \omega)$, if the system (2.2) has an $\rho$-order cosine family $C_{\rho}(t)$ satisfying $(2.4)$.

Lemma 2.8. [21] Let $G: J \times \Omega \rightarrow L_{2}^{0}$ be a strongly measurable mapping such that $\int_{0}^{b} \mathbb{E}\|G(t)\|_{L_{2}^{0}}^{p} d t<\infty$. Then

$$
\mathbb{E}\left\|\int_{0}^{t} G(s) d \omega(s)\right\|^{p} \leq L_{G} \int_{0}^{t} \mathbb{E}\|G(s)\|_{L_{2}^{0}}^{p} d s
$$

for all $t \in J$ and $p \geq 2$, where $L_{G}$ is the constant involving $p$ and $b$.

Definition 2.9. A function $x \in \mathcal{Y}_{2}$ is said to be the mild solution of (2.1) if it satisfies

$$
\begin{aligned}
x(t) & =C_{\rho}(t)\left(x_{0}+g(x)\right)+S_{\rho}(t)\left(x_{1}+g_{1}(x)\right)+\int_{0}^{t} P_{\rho}(t-s)\{B u(s)+\mathfrak{f}(s, x(s))\} d s \\
& +\int_{0}^{t} P_{\rho}(t-s) \sigma(s, x(s)) d \omega(s), t \in[0, b]
\end{aligned}
$$


Definition 2.10. The stochastic system (2.1) is approximately controllable on $[0, b]$ if $\overline{\Re(b)}=L_{2}\left(\Omega, \Im_{b}, \mathcal{Y}\right)$.

Definition 2.11. (Kuratowski measure of noncompactness(MNC)) The Kuratowski measure of noncompactness (MNC) of the set $M$ in the Hilbert space $\mathcal{Y}$ is the greatest lower bound of those $\epsilon>0$ for which the set $M$ can be covered by finitely many sets of diameter $\leq \epsilon$ that is

$\mu(M)=\inf \{\epsilon>0:$ M may be covered by finitely many sets of diameter $\leq \epsilon\}$

for every bounded subset $M$ in the Hilbert space $\mathcal{Y}$.

Lemma 2.12. For any bounded set $U, V \subset \mathcal{Y}$, we have the following results:

1. $\mu(U)=0$ if and only if $U$ is precompact;

2. $\mu(U)=\mu(\operatorname{conv} U)=\mu(\bar{U})$, where convU and $\bar{U}$ denote the convex hull and closure of $U$, respectively;

3. $\mu(U) \subset \mu(V)$, when $U \subset V$;

4. $\mu(U+V) \leq \mu(U)+\mu(V)$, where $U+V=\{u+v: u \in U ; v \in V\}$;

5. $\mu(U \cup V) \leq \max \{\mu(U) ; \mu(V)\}$;

6. $\mu(\lambda U)=\lambda \mu(U)$, for any $\lambda \in \mathbb{R}$;

7. If the map $Q: D(Q) \subset \mathcal{Y} \rightarrow K$ is Lipschitz continuous with constant $\kappa$, then $\mu(Q U) \leq \kappa \mu(U)$ for any bounded subset $U \subset D(Q)$, where $K$ is a Hilbert space.

Definition 2.13. (Condensing Operator) A condensing (or densifying) operator is a mapping under which the image of any set is in a certain sense more compact than the set itself. The degree of noncompactness of a set is measured by means of functions called measures of noncompactness. The contractive maps and the compact maps are condensing.

Theorem 2.14. [23](Sadovskii's Fixed point theorem) Let $N$ be a condensing operator on a Banach space $V$, that is, $N$ is continuous and takes bounded sets into bounded sets, and $\mu(N(D))<\mu(D)$ for every bounded set $D$ of $V$ with $\beta(D)>0$. If $N(S) \subset S$ for a convex, closed and bounded set $S$ of $V$, then $N$ has a fixed point in $S$.(Here $\mu($.$) denotes the Kuratowski measure of non-compactness).$

To prove our main results, we list the following basic assumptions of this paper: 
$\left(H_{1}\right) \quad A$ is the infinitesimal generator of a $\rho$-order cosine family $C_{\rho}(t)$ on $V$ and there exists a constant $M \geq 1$ such that

$$
\left\|C_{\rho}(t)\right\| \leq M
$$

$\left(H_{2}\right)$ The functions $\mathfrak{f}: J \times V \rightarrow V$ and $\sigma: J \times V \rightarrow L_{2}{ }^{0}$ satisfy linear growth and Lipschitz conditions. Moreover, there exist positive constants $N_{1}, N_{2}, K_{1}$ and $K_{2}$ such that

$$
\begin{array}{r}
\|\mathfrak{f}(t, x)-\mathfrak{f}(t, y)\|^{2} \leq N_{1}\|x-y\|^{2}, \quad\|\mathfrak{f}(t, x)\|^{2} \leq N_{2}\left(1+\|x\|^{2}\right) \\
\|\sigma(t, x)-\sigma(t, y)\|_{L_{2}^{0}}^{2} \leq K_{1}\|x-y\|^{2}, \quad\|\sigma(t, x)\|_{L_{2}^{0}}^{2} \leq K_{2}\left(1+\|x\|^{2}\right)
\end{array}
$$

$\left(H_{3}\right)$ The functions $g$ and $g_{1}$ are completely continuous functions and there exist some positive constants $M_{g}$ and $M_{g_{1}}$ such that

$$
\begin{array}{r}
\|g(x)-g(y)\|^{2} \leq M_{g}\|x-y\|^{2}, \quad\|g(x)\|^{2} \leq M_{g}\left(1+\|x\|^{2}\right) \\
\left\|g_{1}(x)-g_{1}(y)\right\|^{2} \leq M_{g_{1}}\|x-y\|^{2}, \quad\left\|g_{1}(x)\right\|^{2} \leq M_{g_{1}}\left(1+\|x\|^{2}\right)
\end{array}
$$

for all $x, y \in C(J, V)$

$\left(H_{4}\right)$ The linear fractional system corresponding to system $(2.1)$ is approximately controllable on $[0, b]$. That is, for each $0 \leq t<b$, the operator $\beta\left(\beta I+\Psi_{t}^{b}\right)^{-1} \rightarrow 0$ in the strong operator topology as $\beta \rightarrow 0^{+}$, where

$$
\Psi_{t}^{b}=\int_{t}^{b} P_{\rho}(b-s) B B^{*} P_{\rho}^{*}(b-s) d s
$$

is the controllability Grammian. It is already known that the linear deterministic system corresponding to $(2.1)$

$$
\left.\begin{array}{rl}
{ }^{c} D_{t}^{\rho} x(t) & =[A x(t)+B u(t)] d t, \quad t \in J \\
x(0) & =x_{0} \text { and } x^{\prime}(0)=x_{1}
\end{array}\right\}
$$

is approximately controllable on $[t, b]$ iff the operator $\beta\left(\beta I+\Psi_{t}^{b}\right)^{-1} \rightarrow 0$ strongly as $\beta \rightarrow 0^{+}[22]$.

\section{MAIN RESULTS}

Let us recall one lemma concerning approximate controllability, which will be used in the proof.

Lemma 3.1. [17] For any $x_{b} \in L_{2}\left(\Omega, \Im_{b}, \mathcal{Y}\right)$, there exists $\phi \in L_{2}^{\Im}\left(J, L_{2}^{0}\right)$ such that $x_{b}=\mathbb{E} x_{b}+\int_{0}^{b} \phi(s) d \omega(s)$. 
Now for any $\beta>0$ and $x_{b} \in L_{2}\left(\Omega, \Im_{b}, \mathcal{Y}\right)$, control function is defined as

$$
\begin{aligned}
u^{\beta}(t, x)= & B^{*} P_{\rho}^{*}(b-t)\left[\left(\beta I+\Psi_{0}^{b}\right)^{-1}\left(\mathbb{E} x_{b}-C_{\rho}(b)\left(x_{0}+g(x)\right)-S_{\rho}(b)\left(x_{1}+g_{1}(x)\right)\right)\right. \\
& \left.+\int_{0}^{t}\left(\beta I+\Psi_{s}^{b}\right)^{-1} \phi(s) d \omega(s)\right] \\
& -B^{*} P_{\rho}^{*}(b-t) \int_{0}^{t}\left(\beta I+\Psi_{s}^{b}\right)^{-1} P_{\rho}(b-s) \mathfrak{f}(s, x(s)) d s \\
& -B^{*} P_{\rho}^{*}(b-t) \int_{0}^{t}\left(\beta I+\Psi_{s}^{b}\right)^{-1} P_{\rho}(b-s) \sigma(s, x(s)) d \omega(s)
\end{aligned}
$$

Lemma 3.2. There exists a positive constant $M_{u}$ such that for all $x, y \in \mathcal{Y}_{2}$, we have

$$
\begin{array}{r}
\mathbb{E}\left\|u^{\beta}(t, x)-u^{\beta}(t, y)\right\|^{2} \leq \frac{M_{u}}{\beta^{2}}\|x-y\|^{2} \\
\mathbb{E}\left\|u^{\beta}(t, x)\right\|^{2} \leq \frac{M_{u}}{\beta^{2}}\left(1+\|x\|^{2}\right)
\end{array}
$$

Proof. Let us take $x, y \in \mathcal{Y}_{2}$. Now, using Holder's inequality, Lemma 1 and the assumed conditions, we get

$$
\begin{aligned}
\mathbb{E} & \left\|u^{\beta}(t, x)-u^{\beta}(t, y)\right\|^{2} \leq 4 \mathbb{E}\left\|B^{*} P_{\rho}^{*}(b-t)\left(\beta I+\Psi_{0}^{b}\right)^{-1} C_{\rho}(b)[g(x)-g(y)]\right\|^{2} \\
& +4 \mathbb{E}\left\|B^{*} P_{\rho}^{*}(b-t)\left(\beta I+\Psi_{0}^{b}\right)^{-1} S_{\rho}(b)\left[g_{1}(x)-g_{1}(y)\right]\right\|^{2} \\
& +4 \mathbb{E}\left\|B^{*} P_{\rho}^{*}(b-t) \int_{0}^{t}\left(\beta I+\Psi_{s}^{b}\right)^{-1} P_{\rho}(b-s)[\mathfrak{f}(s, x(s))-\mathfrak{f}(s, y(s))] d s\right\|^{2} \\
& +4 \mathbb{E}\left\|B^{*} P_{\rho}^{*}(b-t) \int_{0}^{t}\left(\beta I+\Psi_{s}^{b}\right)^{-1} P_{\rho}(b-s)[\sigma(s, x(s))-\sigma(s, y(s))] d \omega(s)\right\|^{2} \\
\leq & \frac{4}{\beta^{2}}\|B\|^{2}\left(\frac{M b^{\rho-1}}{\Gamma(\rho)}\right)^{2} M^{2}\left(M_{g}\|x-y\|_{\mathcal{Y}_{2}}^{2}+M_{g_{1}}\|x-y\|_{\mathcal{Y}_{2}}^{2}\right) \\
& +\frac{4}{\beta^{2}}\|B\|^{2}\left(\frac{M b^{\rho-1}}{\Gamma(\rho)}\right)^{4}\left(b \int_{0}^{t} N_{1} \mathbb{E}\|x(s)-y(s)\|_{\mathcal{Y}}^{2} d s+L_{G} \int_{0}^{t} K_{1} \mathbb{E}\|x(s)-y(s)\|_{\mathcal{Y}}^{2} d s\right) \\
= & \frac{4}{\beta^{2}}\|B\|^{2} M^{4}\left(\frac{b^{\rho-1}}{\Gamma(\rho)}\right)^{2}\left[M_{g}+M_{g_{1}}+\frac{b^{2 \rho}}{\Gamma(\rho)} N_{1} \underset{s \in[0, b]}{\sup } \mathbb{E}\|x(s)-y(s)\|_{\mathcal{Y}}^{2}\right. \\
& \left.+\frac{b^{2 \rho-1}}{\Gamma(\rho)} L_{G} K_{1} \sup _{s \in[0, b]} \mathbb{E}\|x(s)-y(s)\|_{\mathcal{Y}}^{2}\right] \\
\leq & \frac{4}{\beta^{2}}\|B\|^{2} M^{4}\left(\frac{b^{\rho-1}}{\Gamma(\rho)}\right)^{2}\left[M_{g}+M_{g_{1}}+\frac{b^{2 \rho}}{\Gamma(\rho)} N_{1}+\frac{b^{2 \rho-1}}{\Gamma(\rho)} L_{G} K_{1} b\right]\|x-y\|_{\mathcal{Y}_{2}}^{2} \\
= & \frac{M_{u}}{\beta^{2}}\|x-y\|_{\mathcal{Y}_{2}}^{2},
\end{aligned}
$$


where $M_{u}=\frac{4}{\beta^{2}}\|B\|^{2} M^{4}\left(\frac{b^{\rho-1}}{\Gamma(\rho)}\right)^{2}\left[M_{g}+M_{g_{1}}+\frac{b^{2 \rho}}{\Gamma(\rho)} N_{1}+\frac{b^{2 \rho-1}}{\Gamma(\rho)} L_{G} K_{1} b\right]$ and p, q are conjugate indices.

Similarly, we can prove the second inequality. So, the proof of lemma is completed.

For any $\beta>0$, define the operator $\mathbf{F}_{\beta}: \mathcal{Y}_{2} \rightarrow \mathcal{Y}_{2}$ by

$$
\begin{aligned}
\left(\mathbf{F}_{\beta} x\right)(t)=C_{\rho}(t)\left(x_{0}+g(x)\right)+S_{\rho}(t)\left(x_{1}+g_{1}(x)\right)+ & \int_{0}^{t} P_{\rho}(t-s)\left[B u^{\beta}(s, x)+\mathfrak{f}(s, x(s))\right] d s \\
& +\int_{0}^{t} P_{\rho}(t-s) \sigma(s, x(s)) d \omega(s) .
\end{aligned}
$$

To prove the required result, we will use the Sadovskii's Fixed Point theorem to prove the existence of a fixed point of the operator $\mathbf{F}_{\beta}$ defined above in Theorem 3.1. By assuming the approximate controllability of the corresponding deterministic linear system (2.6), we will prove the approximate controllability of system (2.1).

Theorem 3.3. Assume hypothesis $\left(H_{1}\right)-\left(H_{4}\right)$ are satisfied. Then the system (2.1) has a mild solution on $[0, b]$ provided that

$$
10 M^{2}\left(M_{g}+M_{g_{1}}\right)+5\left(\frac{M b^{\rho-1}}{\Gamma(\rho)}\right)^{2}\left(\frac{M_{B}^{2} b^{2} M_{u}}{\beta^{2}}+b^{2} N_{2}+L_{\sigma} K_{2} b\right)<1
$$

and

$$
3\left(\frac{M b^{\rho-1}}{\Gamma(\rho)}\right)^{2}\left(\frac{M_{B}^{2} b M_{u}}{\beta^{2}}+b N_{1}+L_{\sigma}\right)<1 .
$$

Proof. Now the proof is divided into several steps.

Step 1. For any $x \in \mathcal{Y}_{2}, \mathbf{F}_{\beta}(x)(t)$ is continuous on $J$.

Proof of Step 1. Let $0 \leq t_{1} \leq t_{2} \leq b$. Then for any fixed $x \in \mathcal{Y}_{2}$, it follows from Holder's inequality, Lemma 1 and assumptions on the theorem that

$$
\begin{aligned}
& \mathbb{E}\left\|\left(\mathbf{F}_{\beta} x\right)\left(t_{2}\right)-\left(\mathbf{F}_{\beta} x\right)\left(t_{1}\right)\right\|^{2} \leq 8\left\{\mathbb{E}\left\|\left(C_{\rho}\left(t_{2}\right)-C_{\rho}\left(t_{1}\right)\right)\left(x_{0}+g(x)\right)\right\|^{2}\right. \\
& +\mathbb{E}\left\|\left(S_{\rho}\left(t_{2}\right)-S_{\rho}\left(t_{1}\right)\right)\left(x_{1}+g_{1}(x)\right)\right\|^{2} \\
& +\mathbb{E}\left\|\int_{0}^{t_{1}}\left[P_{\rho}\left(t_{2}-s\right)-P_{\rho}\left(t_{1}-s\right)\right] \mathfrak{f}(s, x(s)) d s\right\|^{2} \\
& +\mathbb{E}\left\|\int_{t_{1}}^{t_{2}} P_{\rho}\left(t_{2}-s\right) \mathfrak{f}(s, x(s)) d s\right\|^{2} \\
& +\mathbb{E}\left\|\int_{0}^{t_{1}}\left[P_{\rho}\left(t_{2}-s\right)-P_{\rho}\left(t_{1}-s\right)\right] \sigma(s, x(s)) d \omega(s)\right\|^{2} \\
& +\mathbb{E}\left\|\int_{t_{1}}^{t_{2}} P_{\rho}\left(t_{2}-s\right) \sigma(s, x(s)) d \omega(s)\right\| \|^{2}
\end{aligned}
$$




$$
\begin{aligned}
& +\mathbb{E}\left\|\int_{0}^{t_{1}}\left[P_{\rho}\left(t_{2}-s\right)-P_{\rho}\left(t_{1}-s\right)\right] B u^{\beta}(s, x) d s\right\|^{2} \\
& \left.+\mathbb{E}\left\|\int_{t_{1}}^{t_{2}} P_{\rho}\left(t_{2}-s\right) B u^{\beta}(s, x) d s\right\|^{2}\right\} \\
& \leq 8\left[2 \left(\mathbb{E}\left\|\left(C_{\rho}\left(t_{2}\right)-C_{\rho}\left(t_{1}\right)\right) x_{0}\right\|^{2}+\mathbb{E}\left\|\left(C_{\rho}\left(t_{2}\right)-C_{\rho}\left(t_{1}\right)\right) g(x)\right\|^{2}\right.\right. \\
& +\mathbb{E}\left\|\left(S_{\rho}\left(t_{2}\right)-S_{\rho}\left(t_{1}\right)\right) x_{1}\right\|^{2} \\
& \left.+\mathbb{E}\left\|\left(S_{\rho}\left(t_{2}\right)-S_{\rho}\left(t_{1}\right)\right) g_{1}(x)\right\|^{2}\right)+t_{1} \int_{0}^{t_{1}} \mathbb{E}\left\|\left[P_{\rho}\left(t_{2}-s\right)-P_{\rho}\left(t_{1}-s\right]\right) \mathfrak{f}(s, x(s))\right\|^{2} d s \\
& +\left(\frac{M b^{\rho-1}}{\Gamma(\rho)}\right)^{2}\left(t_{2}-t_{1}\right) \int_{t_{1}}^{t_{2}} \mathbb{E}\|\mathfrak{f}(s, x(s))\|^{2} d s \\
& +L_{G} \int_{0}^{t_{1}} \mathbb{E}\left\|\left(P_{\rho}\left(t_{2}-s\right)-P_{\rho}\left(t_{1}-s\right)\right) \sigma(s, x(s))\right\|^{2} d s \\
& +\left(\frac{M b^{\rho-1}}{\Gamma(\rho)}\right)^{2} L_{G} \int_{t_{1}}^{t_{2}} \mathbb{E}\|\sigma(s, x(s))\|^{2} d s \\
& +t_{1} \int_{0}^{t_{1}} \mathbb{E}\left\|\left[P_{\rho}\left(t_{2}-s\right)-P_{\rho}\left(t_{1}-s\right)\right] B u^{\beta}(s, x)\right\|^{2} d s \\
& +\|B\|^{2}\left(\frac{M b^{\rho-1}}{\Gamma(\rho)}\right)^{2}\left(t_{2}-t_{1}\right) \int_{t_{1}}^{t_{2}} \mathbb{E}\left\|u^{\beta}(s, x)\right\|^{2} d s .
\end{aligned}
$$

Since $C_{\rho}(t), S_{\rho}(t)$ and $P_{\rho}(t)$ are strongly continuous. So, using Lebesgue's dominated convergence theorem, we get that $\mathbf{F}_{\beta}(x)(t)$ is continuous on $J$.

Step 2. For each positive integer $q$, let $B_{q}=\left\{x \in \mathcal{Y}_{2}: \mathbb{E}\|x(t)\|_{\mathcal{Y}}^{2} \leq q\right\}$, then the set $B_{q}$ is clearly a bounded, closed and convex set in $\mathcal{Y}_{2}$. Using Holder's inequality and assumption (ii), we derive that

$$
\begin{aligned}
\mathbb{E}\left\|\int_{0}^{t} P_{\rho}(t-s) \mathfrak{f}(s, x(s)) d s\right\|_{\mathcal{Y}}^{2} & \leq \mathbb{E}\left(\int_{0}^{t}\left\|P_{\rho}(t-s) \mathfrak{f}(s, x(s))\right\|_{\mathcal{Y}} d s\right)^{2} \\
& \leq\left(\frac{M b^{\rho-1}}{\Gamma(\rho)}\right)^{2} \mathbb{E}\left(\int_{0}^{t}\|\mathfrak{f}(s, x(s))\|_{\mathcal{Y}} d s\right)^{2} \\
& \leq\left(\frac{M b^{\rho-1}}{\Gamma(\rho)}\right)^{2} b \int_{0}^{t} \mathbb{E}\|\mathfrak{f}(s, x(s))\|_{\mathcal{Y}}^{2} d s \\
& \leq\left(\frac{M b^{\rho-1}}{\Gamma(\rho)}\right)^{2} b \int_{0}^{t} N_{2}\left(1+\mathbb{E}\|x(s)\|_{\mathcal{Y}}^{2}\right) d s \\
& \leq\left(\frac{M b^{\rho-1}}{\Gamma(\rho)}\right)^{2} b N_{2} \int_{0}^{t}\left(1+\sup _{s \in[0, b]} \mathbb{E}\|x(s)\|_{\mathcal{Y}}^{2}\right) d s \\
& \leq\left(\frac{M b^{\rho-1}}{\Gamma(\rho)}\right)^{2} b N_{2} b\left(1+\|x\|_{\mathcal{Y}_{2}}^{2}\right) \\
& \leq\left(\frac{M b^{\rho-1}}{\Gamma(\rho)}\right)^{2} b^{2} N_{2}\left(1+\|x\|_{\mathcal{Y}_{2}}^{2}\right)
\end{aligned}
$$


Which deduces that $P_{\rho}(t-s) \mathfrak{f}(s, x(s))$ is integrable on $J$, by Bochner's Theorem [18], $\mathbf{F}_{\beta}$ is well defined on $B_{q}$.

Similarly using lemma 2.8 and assumption $(i i)$, we derive that

$$
\begin{aligned}
\mathbb{E}\left\|\int_{0}^{t} P_{\rho}(t-s) \sigma(s, x(s)) d w(s)\right\|^{2} & \leq L_{\sigma} \int_{0}^{t} \mathbb{E}\left\|P_{\rho}(t-s) \sigma(s, x(s))\right\|_{L_{2}^{0}}^{2} d s \\
& \leq L_{\sigma}\left(\frac{M b^{\rho-1}}{\Gamma(\rho)}\right)^{2} \int_{0}^{t} \mathbb{E}\|\sigma(s, x(s))\|_{L_{2}^{0}}^{2} d s \\
& \leq L_{\sigma}\left(\frac{M b^{\rho-1}}{\Gamma(\rho)}\right)^{2} \int_{0}^{t} K_{2}\left(1+\mathbb{E}\|x(s)\|_{\mathcal{Y}}^{2}\right) d s \\
& \leq L_{\sigma}\left(\frac{M b^{\rho-1}}{\Gamma(\rho)}\right)^{2} K_{2} \int_{0}^{t}\left(1+\sup _{s \in[0, b]} \mathbb{E}\|x(s)\|_{\mathcal{Y}}^{2}\right) d s \\
& \leq L_{\sigma}\left(\frac{M b^{\rho-1}}{\Gamma(\rho)}\right)^{2} K_{2} b\left(1+\|x\|_{\mathcal{Y}_{2}}^{2}\right) \\
& \leq L_{\sigma}\left(\frac{M b^{\rho-1}}{\Gamma(\rho)}\right)^{2} K_{2} b\left(1+\|x\|_{\mathcal{Y}_{2}}^{2}\right)
\end{aligned}
$$

Now, we claim that there exists a positive number $q$ such that $\mathbf{F}_{\beta}\left(B_{q}\right) \subseteq B_{q}$.

If it is not true, then for each positive number $q$, there is a function $x_{q}(.) \in B_{q}$ but $\mathbf{F}_{\beta} x_{q}$ doesnot belong to $B_{q}$, that is $\mathbb{E}\left\|\mathbf{F}_{\beta} x_{q}(t)\right\|_{\mathcal{Y}}^{2}>q$ for some $t \in J$.

On the other hand, from assumptions (ii),(iii) and lemma 1, we have

$$
\begin{aligned}
q & \leq \mathbb{E}\left\|F_{\beta} x_{q}(t)\right\|_{\mathcal{Y}}^{2}=\mathbb{E} \| C_{\rho}(t)\left(x_{0}+g(x)\right)+S_{\rho}(t)\left(x_{1}+g_{1}(x)\right)+\int_{0}^{t} P_{\rho}(t-s)\left[B u^{\beta}(s, x)\right. \\
& +\mathfrak{f}(s, x(s))] d s+\int_{0}^{t} P_{\rho}(t-s) \sigma(s, x(s)) d w(s) \|_{\mathcal{Y}}^{2} \\
\leq & 5 M^{2} \mathbb{E}\left\|x_{0}+g(x)\right\|^{2}+5 M^{2} \mathbb{E}\left\|x_{1}+g_{1}(x)\right\|^{2} \\
& +5\left(\frac{M b^{\rho-1}}{\Gamma(\rho)}\right)^{2} M_{B}^{2} b^{2} \frac{M_{u}}{\beta^{2}}\left(1+\|x\|_{\mathcal{Y}_{2}}^{2}\right)+5\left(\frac{M b^{\rho-1}}{\Gamma(\rho)}\right)^{2} b^{2} N_{2}\left(1+\|x\|_{\mathcal{Y}_{2}}^{2}\right) \\
& +5\left(\frac{M b^{\rho-1}}{\Gamma(\rho)}\right)^{2} L_{\sigma} K_{2} b\left(1+\|x\|_{\mathcal{Y}_{2}}^{2}\right) \\
\leq & M^{2}\left(2 \mathbb{E}\left\|x_{0}\right\|^{2}+2 \mathbb{E}\|g(x)\|^{2}\right)+5 M^{2}\left(2 \mathbb{E}\left\|x_{1}\right\|^{2}+2 \mathbb{E}\left\|g_{1}(x)\right\|^{2}\right) \\
& +5\left(\frac{M b^{\rho-1}}{\Gamma(\rho)}\right)^{2} M_{B}^{2} b^{2} \frac{M_{u}}{\beta^{2}}\left(1+\|x\|_{\mathcal{Y}_{2}}^{2}\right)+5\left(\frac{M b^{\rho-1}}{\Gamma(\rho)}\right)^{2} b^{2} N_{2}\left(1+\|x\|_{\mathcal{Y}_{2}}^{2}\right) \\
& +5\left(\frac{M b^{\rho-1}}{\Gamma(\rho)}\right)^{2} L_{\sigma} K_{2} b\left(1+\|x\|_{\mathcal{Y}_{2}}^{2}\right) \\
\leq & M^{2}\left[2 \mathbb{E}\left\|x_{0}\right\|^{2}+2 M_{g}\left(1+\|x\|_{\mathcal{Y}_{2}}^{2}\right)\right]+5 M^{2}\left[2 \mathbb{E}\left\|x_{1}\right\|^{2}+2 M_{g_{1}}\left(1+\|x\|_{\mathcal{Y}_{2}}^{2}\right)\right] \\
& +5\left(\frac{M b^{\rho-1}}{\Gamma(\rho)}\right)^{2} M_{B}^{2} b^{2} \frac{M_{u}}{\beta^{2}}\left(1+\|x\|_{\mathcal{Y}_{2}}^{2}\right)+5\left(\frac{M b^{\rho-1}}{\Gamma(\rho)}\right)^{2} b^{2} N_{2}\left(1+\|x\|_{\mathcal{Y}_{2}}^{2}\right)
\end{aligned}
$$




$$
\begin{aligned}
& +5\left(\frac{M b^{\rho-1}}{\Gamma(\rho)}\right)^{2} L_{\sigma} K_{2} b\left(1+\|x\|_{\mathcal{Y}_{2}}^{2}\right) \\
\leq & 5 M^{2}\left[2 \mathbb{E}\left\|x_{0}\right\|^{2}+2 M_{g}(1+q)\right]+5 M^{2}\left[2 \mathbb{E}\left\|x_{1}\right\|^{2}+2 M_{g_{1}}(1+q)\right] \\
& +5\left(\frac{M b^{\rho-1}}{\Gamma(\rho)}\right)^{2} M_{B}^{2} b^{2} \frac{M_{u}}{\beta^{2}}(1+q)+5\left(\frac{M b^{\rho-1}}{\Gamma(\rho)}\right)^{2} b^{2} N_{2}(1+q) \\
& +5\left(\frac{M b^{\rho-1}}{\Gamma(\rho)}\right)^{2} L_{\sigma} K_{2} b(1+q) \\
\leq & {\left[10 M^{2} \mathbb{E}\left\|x_{0}\right\|^{2}+10 M^{2} M_{g}+10 M^{2} \mathbb{E}\left\|x_{1}\right\|^{2}+10 M^{2} M_{g_{1}}\right.} \\
& \left.+5\left(\frac{M b^{\rho-1}}{\Gamma(\rho)}\right)^{2}\left(\frac{M_{B}^{2} b^{2} M_{u}}{\beta^{2}}+b^{2} N_{2}+L_{\sigma} K_{2} b\right)\right]+\left[10 M^{2} M_{g}\right. \\
& \left.+10 M^{2} M_{g_{1}}+5\left(\frac{M b^{\rho-1}}{\Gamma(\rho)}\right)^{2}\left(\frac{M_{B}^{2} b^{2} M_{u}}{\beta^{2}}+b^{2} N_{2}+L_{\sigma} K_{2} b\right)\right] q .
\end{aligned}
$$

Dividing both sides by $q$ and taking the limit as $q \rightarrow \infty$, we get

$$
10 M^{2}\left(M_{g}+M_{g_{1}}\right)+5\left(\frac{M b^{\rho-1}}{\Gamma(\rho)}\right)^{2}\left(\frac{M_{B}^{2} b^{2} M_{u}}{\beta^{2}}+b^{2} N_{2}+L_{\sigma} K_{2} b\right)>1
$$

This contradicts condition (3.3). Hence for some positive number $q, \mathbf{F}_{\beta} B_{q} \subseteq B_{q}$.

Step 3. Now, we define operators $\mathbf{F}_{\beta_{1}}$ and $P_{\beta_{2}}$ as

$$
\begin{aligned}
& \left(\mathbf{F}_{\beta_{1}} x\right)(t)=C_{\rho}(t)\left[x_{0}+g(x)\right]+S_{\rho}(t)\left[x_{1}+g_{1}(x)\right] \\
& \left(\mathbf{F}_{\beta_{2}} x\right)(t)=\int_{0}^{t} P_{\rho}(t-s)\left[B u^{\beta}(s, x)+\mathfrak{f}(s, x(s))\right] d s+\int_{0}^{t} P_{\rho}(t-s) \sigma(s, x(s)) d \omega(s)
\end{aligned}
$$

for $t \in J$.

Here, we will prove that $\mathbf{F}_{\beta_{1}}$ is completely continuous, while $\mathbf{F}_{\beta_{2}}$ is a contraction operator.

By assumption (iii), it is clear that $\mathbf{F}_{\beta_{1}}$ is a completely continuous operator.

Next we show that $\mathbf{F}_{\beta_{2}}$ is the contraction operator. For this, let $x, y \in B_{q}$, then for each $t \in J$, we have from assumption $(i i)$ and Lemma $4 \mathbb{E}\left\|\left(\mathbf{F}_{\beta_{2}} x\right)(t)-\left(\mathbf{F}_{\beta_{2}} y\right)(t)\right\|_{\mathcal{Y}}^{2}$

$$
\begin{aligned}
\leq & 3 \mathbb{E}\left\|\int_{0}^{t} P_{\rho}(t-s) B\left[u^{\beta}(s, x)-u^{\beta}(s, y)\right] d s\right\|_{\mathcal{Y}}^{2} \\
& +3 \mathbb{E}\left\|\int_{0}^{t} P_{\rho}(t-s)[\mathfrak{f}(s, x(s))-\mathfrak{f}(s, y(s))] d s\right\|_{\mathcal{Y}}^{2} \\
& +3 \mathbb{E}\left\|\int_{0}^{t} P_{\rho}(t-s)[\sigma(s, x(s))-\sigma(s, y(s))] d \omega(s)\right\|_{\mathcal{Y}}^{2} \\
\leq & 3\left(\frac{M b^{\rho-1}}{\Gamma(\rho)}\right)^{2} M_{B}^{2} \int_{0}^{t} \mathbb{E}\left\|u^{\beta}(s, x)-u^{\beta}(s, y)\right\|_{\mathcal{Y}}^{2} d s \\
& +3\left(\frac{M b^{\rho-1}}{\Gamma(\rho)}\right)^{2} \int_{0}^{t} \mathbb{E}\|\mathfrak{f}(s, x(s))-\mathfrak{f}(s, y(s))\|^{2} d s
\end{aligned}
$$




$$
\begin{aligned}
& +3\left(\frac{M b^{\rho-1}}{\Gamma(\rho)}\right)^{2} \int_{0}^{t} \mathbb{E}\|\sigma(s, x(s))-\sigma(s, y(s))\|^{2} d w(s) \\
\leq & 3\left(\frac{M b^{\rho-1}}{\Gamma(\rho)}\right)^{2} M_{B}^{2} b \frac{M_{u}}{\beta^{2}}\|x-y\|_{\mathcal{Y}_{2}}^{2}+3\left(\frac{M b^{\rho-1}}{\Gamma(\rho)}\right)^{2} b N_{1}\|x-y\|_{\mathcal{Y}_{2}}^{2} \\
& +3\left(\frac{M b^{\rho-1}}{\Gamma(\rho)}\right)^{2} L_{\sigma}\|x-y\|_{\mathcal{Y}_{2}}^{2} \\
\leq & 3\left(\frac{M b^{\rho-1}}{\Gamma(\rho)}\right)^{2}\left(\frac{M_{B}^{2} b M_{u}}{\beta^{2}}+b N_{1}+L_{\sigma}\right)\|x-y\|_{\mathcal{Y}_{2}}^{2}
\end{aligned}
$$

Taking supremum over $t \in[0, b]$, we get

$$
\sup _{t \in[0, b]} \mathbb{E}\left\|\left(\mathbf{F}_{\beta_{2}} x\right)(t)-\left(\mathbf{F}_{\beta_{2}} y\right)(t)\right\|_{\mathcal{Y}}^{2} \leq L_{0}\|x-y\|_{\mathcal{Y}_{2}}^{2}
$$

therefore

$$
\left\|\left(\mathbf{F}_{\beta_{2}} x\right)-\left(\mathbf{F}_{\beta_{2}} y\right)\right\|_{\mathcal{Y}_{2}}^{2} \leq L_{0}\|x-y\|_{\mathcal{Y}_{2}}^{2}
$$

where

$$
L_{0}=3\left(\frac{M b^{\rho-1}}{\Gamma(\rho)}\right)^{2}\left(\frac{M_{B}^{2} b M_{u}}{\beta^{2}}+b N_{1}+L_{\sigma}\right)<1
$$

Thus $\mathbf{F}_{\beta_{2}}$ is a contraction mapping.

Now we have $\mathbf{F}_{\beta}=\mathbf{F}_{\beta_{1}}+\mathbf{F}_{\beta_{2}}$ is a condensing map on $B_{q}$, so all the conditions of Sadovskii's Fixed Point theorem are satisfied. Hence we conclude that there exists a fixed point $x($.$) for \mathbf{F}_{\beta}$ on $B_{q}$, which is the mild solution of (2.1).

Theorem 3.4. Assume that $\left(H_{1}\right)-\left(H_{3}\right)$ hold and $\left\{P_{\rho}(t): t \geq 0\right\}$ is compact. Moreover, if $\mathfrak{f}$ and $\sigma$ are uniformly bounded, then the system (2.1) is approximately controllable on $[0, b]$.

Proof. By Theorem 3.1, $\mathbf{F}_{\beta}$ has a unique fixed point say $x_{\beta}$ in $\mathcal{Y}_{2}$. Now, we can show using stochastic Fubini Theorem that $x_{\beta}$ satisfies

$$
\begin{aligned}
x_{\beta}(b) & =x_{b}-\beta\left(\beta I+\Psi_{o}^{b}\right)^{-1}\left(\mathbb{E} x_{b}-C_{\rho}(b)\left(x_{0}+g(x)\right)-S_{\rho}(b)\left(x_{1}+g_{1}(x)\right)\right) \\
& +\beta \int_{0}^{b}\left(\beta I+\Psi_{s}^{b}\right)^{-1} P_{\rho}(b-s) \mathfrak{f}\left(s, x_{\beta}(s)\right) d s \\
& +\beta \int_{0}^{b}\left(\beta I+\Psi_{s}^{b}\right)^{-1}\left[P_{\rho}(b-s) \sigma\left(s, x_{\beta}(s)\right)-\phi(s)\right] d \omega(s)
\end{aligned}
$$

Using the uniform boundedness of $\mathfrak{f}$ and $\sigma$, there exists $D>0$ such that

$$
\left\|\mathfrak{f}\left(s, x_{\beta}(s)\right)\right\|^{2}+\left\|\sigma\left(s, x_{\beta}(s)\right)\right\|^{2} \leq D
$$

in $[0, b] \times \Omega$. Then we have a subsequence denoted by $\left\{\mathfrak{f}\left(s, x_{\beta}(s)\right), \sigma\left(s, x_{\beta}(s)\right)\right\}$ weakly converging to say $\{\mathfrak{f}(s, \omega), \sigma(s, \omega)\}$ in $\mathcal{Y} \times L_{2}^{0}$. Using the compactness of $P_{\rho}(t)$, we get $P_{\rho}(b-s) \mathfrak{f}\left(s, x_{\beta}(s)\right) \rightarrow P_{\rho}(b-s) \mathfrak{f}(s) ; P_{\rho}(b-s) \sigma\left(s, x_{\beta}(s)\right) \rightarrow P_{\rho}(b-s) \sigma(s)$ in $J \times \Omega$. 
Now, from the above equation, we get

$$
\begin{aligned}
\mathbb{E}\left\|x_{\beta}(b)-x_{b}\right\|^{2} \leq & 6\left\|\beta\left(\beta I+\Psi_{0}^{b}\right)^{-1}\left[\mathbb{E} x_{b}-C_{\rho}(b)\left(x_{0}+g(x)\right)-S_{\rho}(b)\left(x_{1}+g_{1}(x)\right)\right]\right\|^{2} \\
& +6 \mathbb{E}\left(\int_{0}^{b}\left\|\beta\left(\beta I+\Psi_{s}^{b}\right)^{-1} \phi(s)\right\|_{L_{2}^{0}}^{2} d s\right) \\
& +6 \mathbb{E}\left(\int_{0}^{b}\left\|\beta\left(\beta I+\Psi_{s}^{b}\right)^{-1}\right\|\left\|P_{\rho}(b-s)\left[\mathfrak{f}\left(s, x_{\beta}(s)\right)-\mathfrak{f}(s)\right]\right\| d s\right)^{2} \\
& +6 \mathbb{E}\left(\int_{0}^{b}\left\|\beta\left(\beta I+\Psi_{s}^{b}\right)^{-1} P_{\rho}(b-s) \mathfrak{f}(s)\right\| d s\right)^{2} \\
& +6 \mathbb{E}\left(\int_{0}^{b}\left\|\beta\left(\beta I+\Psi_{s}^{b}\right)^{-1}\right\|\left\|P_{\rho}(b-s)\left[\sigma\left(s, x_{\beta}(s)\right)-\sigma(s)\right]\right\|_{L_{2}^{0}}^{2} d s\right) \\
& \left.+6 \mathbb{E}\left(\int_{0}^{b}\left\|\beta\left(\beta I+\Gamma_{s}^{b}\right)^{-1} P_{\rho}(b-s) \sigma(s)\right\|_{L_{2}^{0}}^{2} d s\right)\right]
\end{aligned}
$$

since by assumption $\left(H_{4}\right)$, for all $0 \leq s<b$ the operator $\beta\left(\beta I+\Psi_{s}^{b}\right)^{-1} \rightarrow 0$ strongly as $\beta \rightarrow 0^{+}$and $\left\|\beta\left(\beta I+\Psi_{s}^{b}\right)^{-1}\right\| \leq 1$. Thus by the Lebesgue dominated convergence theorem, we obtain $\mathbb{E}\left\|x_{\beta}(b)-x_{b}\right\|^{2} \rightarrow 0^{+}$. This gives the approximate controllability of system (2.1).

\section{EXAMPLE}

Let us consider the control system governed by the following partial differential equation:

$$
\begin{aligned}
& { }^{C} D_{t}^{\rho} y(t, x)=y_{x x}(t, x)+\mu(t, x)+\mathfrak{f}(t, y(t))+\sigma(t, x(t)) \frac{d \omega(t)}{d t} ; t \in[0, b] \\
& y(t, 0)=y(t, \pi)=0 ; \text { for } t \in[0, b] \\
& y(0, x)+\sum_{i=1}^{n} \alpha_{i} y\left(t_{i}, x\right)=y_{0}(x), \quad t \in J \\
& y_{t}(0, x)+\sum_{i=1}^{k} \beta_{i} y\left(s_{i}, x\right)=y_{1}(x)
\end{aligned}
$$

where $\rho \in(1,2]$, one can take arbitrary nonlinear function $\mathfrak{f}$ satisfying the condition $\left(H_{2}\right)$. Let $\mathcal{Y}=L_{2}[0, \pi]$ be as in introduction. Let control function $\mu:[0, b] \times(0, \pi) \rightarrow \mathbb{R}$ be continuous in $t$.

Define $A: D(A) \subseteq \mathcal{Y} \rightarrow \mathcal{Y}$ by

$$
A w=w^{\prime \prime} ; \quad w \in D(A)
$$

where $D(A)=\left\{w \in \mathcal{Y}: w, w^{\prime}\right.$ are absolutely continuous, $\left.w^{\prime \prime} \in \mathcal{Y}, w(0)=w(\pi)=0\right\}$. 
Then, $A$ has the spectral representation

$$
A w=\sum_{n=1}^{+\infty}-n^{2}\left(w, w_{n}\right) w_{n}, \quad w \in D(A)
$$

where $w_{n}(s)=\sqrt{\frac{2}{\pi}} \sin n s, n=1,2,3, \ldots$ is the orthogonal set of eigenfunctions of $A$. $A$ is the infinitesimal generator of a strongly continuous cosine family $\{C(t): t \in \mathbb{R}\}$, defined on $\mathcal{Y}$ which is given by

$$
C(t) w=\sum_{n=1}^{+\infty} \cos n t\left(w, w_{n}\right) w_{n}, \quad w \in \mathcal{Y},
$$

and, the associated sine family is given by

$$
S(t) w=\sum_{n=1}^{+\infty} \frac{1}{n} \sin n t\left(w, w_{n}\right) w_{n}, \quad w \in \mathcal{Y},
$$

Let the control operator $B u:[0, b] \rightarrow \mathcal{Y}$ defined by

$$
(B u)(t)(y)=\mu(t, y) ; \quad y \in(0, \pi) .
$$

For $\rho \in(1,2]$, since $A$ is the infinitesimal generator of a strongly continuous cosine family $C(t)$, from the subordinate principle [2], it follows that $A$ is the infinitesimal generator of a strongly continuous exponentially bounded fractional cosine family $C_{\rho}(t)$ such that $C_{\rho}(0)=I$, and

$$
C_{\rho}(t)=\int_{0}^{\infty} \varphi_{t, \rho / 2}(s) C(s) d s, t>0
$$

where $\varphi_{t, \rho / 2}(s)=t^{-\rho / 2} \phi_{\rho / 2}\left(s t^{-\rho / 2}\right)$, and

$$
\phi_{\gamma}(x)=\sum_{n=0}^{\infty} \frac{(-x)^{n}}{n ! \Gamma(-\gamma n+1-\gamma)}, \quad 0<\gamma<1 .
$$

The functions $g, g_{1}: C(J, \mathcal{Y}) \rightarrow \mathcal{Y}$ is defined as

$$
g(y)(x)=\sum_{i=1}^{n} \alpha_{i} y\left(t_{i}, x\right) \text { and } g_{1}(y)(x)=\sum_{i=1}^{k} \beta_{i} y\left(s_{i}, x\right)
$$

for $0<t_{i}, s_{i}<b$ and $x \in[0, \pi]$.

The problem (4.1) can be rewritten as

$$
\begin{aligned}
{ }^{C} D_{t}^{\rho} y(t) & =A y(t)+B u(t)+\mathfrak{f}(t, x(t))+\sigma(t, x(t)) \frac{d \omega}{d t} ; t \in[0, b] \\
y(0) & =y_{0}+g(y) \\
y^{\prime}(0) & =y_{1}+g_{1}(y)
\end{aligned}
$$

Therefore, by Theorem 3.1, if the hypothesis $\left(H_{1}\right)-\left(H_{4}\right)$ are satisfied, the differential system (4.1) is approximately controllable on $[0, b]$. 


\section{REFERENCES}

[1] U. Arora, Sukavanam, N. Sukavanam, Approximate controllability of second order semilinear stochastic system with nonlocal conditions, Appl. Math. Comput., 258 2015, 111-119.

[2] E.G. Bajlekova, Fractional Evolution Equations in Banach Spaces, Eindhoven University of Technology, Eindhoven, 2001.

[3] Stephen Barnett, Introduction to Mathematical Control Theory, Clarendon press, Oxford 1975.

[4] L. Byszewski and V. Lakshmikantham, Theorem about the existence and uniqueness of a solution of a nonlocal abstract Cauchy problem in a Banach space, Appl. Anal., 40 (1991), no. 1, 11-19.

[5] Y. K. Chang, J. J. Nieto and W. S. Li, Controllability of semilinear differential systems with nonlocal initial conditions in Banach spaces, J. Optim. Theory Appl., 142 (2009), no. 2, 267-273.

[6] R. F. Curtain and H. Zwart, An introduction to infinite-dimensional linear systems theory, Texts in Applied Mathematics, 21, Springer, New York, 1995.

[7] S. Farahi and T. Guendouzi, Approximate controllability of fractional neutral stochastic evolution equations with nonlocal conditions, Results Math., 65 (2014), no. 3-4, 501-521.

[8] T. Guendouzi and S. Farahi, Approximate controllability of semilinear fractional stochastic dynamic systems with nonlocal conditions in Hilbert spaces, Mediterr. J. Math., 13 (2016), no. 2, 637-656.

[9] R.E. Kalman, Controllability of linear systems, Contributions to Differential Equations, 1 (1963), 190-213.

[10] A.A. Kilbas, H.M. Srivastava and J.J. Trujillo, Theory and applications of fractional differential equations, North-Holland Mathematics Studies, 204, Elsevier, Amsterdam, 2006.

[11] J. Klamka, Stochastic controllability of linear systems with delay in contol, Bulletin of the Polish Academy of Sciences, 55 (2007), 23-29.

[12] J. Klamka and L. Socha, Some remarks about stochastic controllability, IEEE Trans. Automatic Control, AC-22 (1977), no. 5, 880-881.

[13] S. Kumar and N. Sukavanam, Controllability of second-order systems with nonlocal conditions in Banach spaces, Numer. Funct. Anal. Optim., 35 (2014), no. $4,423-431$. 
[14] A. Shukla, Urvashi Arora and N. Sukavanam, Approximate controllability of retarded semilinear stochastic system with non local conditions, J. Appl. Math. Comput., doi: 10.1007/s12190-014-0851-9.

[15] L. Shen and J. Sun, Relative controllability of stochastic nonlinear systems with delay in control, Nonlinear Anal. Real World Appl., 13 (2012), no. 6, 2880-2887.

[16] N.I. Mahmudov, Approximate controllability of semilinear deterministic and stochastic evolution equations in abstract spaces, SIAM J. Control Optim., 42 (2003), no. 5, 1604-1622.

[17] N.I. Mahmudov, Controllability of linear stochastic systems in Hilbert spaces, $J$. Math. Anal. Appl., 259 (2001), no. 1, 64-82.

[18] C.-M. Marle, Mesures et probabilités, Hermann, Paris, 1974.

[19] K.S. Miller and B. Ross, An Introduction to the Fractional Calculus and Fractional Differential Equations, A Wiley-Interscience Publication, Wiley, New York, 1993.

[20] I. Podlubny, Fractional Differential Equations, Mathematics in Science and Engineering, 198, Academic Press, San Diego, CA, 1999.

[21] G. Da Prato and J. Zabczyk, Stochastic Equations in Infinite Dimensions, Encyclopedia of Mathematics and its Applications, 44, Cambridge Univ. Press, Cambridge, 1992.

[22] R. Sakthivel, Y. Ren and N.I. Mahmudov, On the approximate controllability of semilinear fractional differential systems, Comput. Math. Appl., 62 (2011), no. 3, 1451-1459.

[23] E. Zeidler, Nonlinear Functional Analysis and its Applications, I, Translated from the German by Peter R. Wadsack, Springer, New York, 1986. 